\title{
REFLECTIONS ON SOROS:
}

\section{MACH, QUINE, ARTHUR AND FAR-FROM-EQUILIBRIUM DYNAMICS}

\author{
Rod Cross ${ }^{1}$ \\ Harold Hutchinson ${ }^{2}$ \\ Harbir Lamba ${ }^{3}$ \\ Doug Strachan ${ }^{4}$
}

$29^{\text {th }}$ July 2013

This paper is an invited submission to the Journal of Economic Methodology Symposium on Reflexivity.

ABSTRACT: We argue that the Soros account of reflexivity does not provide a clear-cut distinction between a social science such as economics and the physical sciences. It is pointed out that the participants who attempt to learn from refutations of conjectures in the Soros world are likely to be haunted by the Duhem-Quine problem of conjointness of hypotheses and unfocussed refutation. On a more constructive note, we argue that models of inductive learning, in which participants form conjectures on the basis of strictly limited information sets, can capture the basic thrust of the Soros position. The conjectures are in motion, as the participants attempt to avoid those that are systematically wrong, and there is something vague and uncertain about what can be learned from experience and refutations. The only notion of market efficiency in this world is one contingent on the strictly limited and varied information sets in play. Finally we present a mathematical model and numerical simulations that help justify the causal relationship between reflexivity and far-from-equilibrium dynamics postulated by Soros.

JEL CLASSIFICATION: B41, A12, C60, G10.

KEYWORDS: Soros, Reflexivity, Far-from-Equilibrium, Mach, Quine, El Farol.

1. Corresponding author: Department of Economics, University of Strathclyde, Glasgow, UK, G4 0GE. rod.cross@strath.ac.uk

2. Investec Bank plc.

3. Department of Mathematical Sciences, George Mason University, Fairfax, VA, USA, 22030.

4. Department of Economics, University of Strathclyde.

We are most grateful for the comments and assistance of Michael Grinfeld and Mrs Irene Spencer in the preparation of this paper. 
It is not uncommon to hear those engaged in business or financial markets say that economic theories might be OK in theory, but not in practice. Not many of these practitioners open up a dialogue by saying precisely what is wrong with the theories in question, and how the theories might be reformulated to improve their alignment with practice. George Soros is a notable exception. "Fallibility, Reflexivity and the Human Uncertainty Principle" [1] is a valuable addition to his earlier contributions to such a dialogue.

In what follows we will attempt to be constructive in relation to aspects of the Soros position that we have selected for attention. A first section deals with the Soros guillotine between the physical and social sciences, considering the treatment of this issue by Mach [2]. Then we comment on the Popperian perspective underlying the views of Soros, using the lens of Quine [3]. A third section deals with the learning problems raised by the Soros position, using the El Farol bar attendance parable of Arthur[4] as a vehicle for exposition. The following section considers the implications of the Baddeley [6] concept of working memory for the Soros critique of the rational expectations and efficient markets hypotheses. The final section uses the heterogeneous agent model of Lamba $[19,20]$ (following from Cross, Grinfeld, Lamba and Seaman [5]) to help clarify how far-from-equilibrium behaviour in the Soros world arises from the non-standard elements of reflexivity.

\section{The Soros Guillotine}

Soros argues that there is a dichotomy between the physical and social sciences because the latter deal with decisions made by thinking participants. "In natural science the outside observer is engaged only in the cognitive function... By contrast, in human affairs, thinking is part of the subject matter. The course of events leads not only from facts to facts but also from facts to the participants' perceptions (the cognitive function) and from the participants' decisions to facts (the manipulative function)." [1, p.17]. A human uncertainty principle arises from it not being possible for participants in social systems to know what other participants are thinking, what their interests and values are, and so on. Reflexivity is taken to involve a two-way process whereby reality helps determine participants' views, and vice versa.

This Soros guillotine between the physical and social sciences relies on there being a compelling logic underlying scientific method that can be applied to the physical sciences. For Soros it is taken as an article of faith that Popper's falsificationist account of science holds. To uphold an account of science in terms of a logic of scientific method requires a clear distinction between theories and observational evidence. The Popper version of hypothetico-deductive logic is $H \rightarrow I . O \rightarrow H$, where $H$ stands for "hypothesis", $\rightarrow$ indicates "implies", . indicates "conjoined with", I stands for "implications", indicates "not", and 0 stands for "observational evidence". Thus a hypothesis is demonstrated to be false if the observational evidence is inconsistent with the implications of the hypothesis.

In the Soros account, theories are independent of observational evidence in the physical but not in the social world. This is because of reflexivity, by way of the manipulative 
function of thought, in the social world. "As we have seen, natural phenomena provide a genuinely independent criterion for judging the validity of generalizations relating to them, but the facts produced by social processes do not do so because they are influenced by theories held by participants." $[1$, p.20].

Alternative accounts of the physical sciences do not rely on there being a compelling logic underlying scientific method, and involve something similar to the two-way interaction between theories and their subject matter that Soros takes to be confined to the social world. Mach's account of science is instructive in this respect. His work on physics is well known [2], but he was also a psychologist, working on the physiology of sensations [7], and so was well placed to formulate a philosophy of science that could encompass, if appropriate, the physical and human worlds. Samuelson, a towering figure in economics in the second half of the $20^{\text {th }}$ century, deemed Mach's conventionalist account of science to be that most consonant with good practice in economics [8], and so is at least worthy of consideration [9].

The starting point for Mach is one of reality being a non-repeating mosaic of elemental qualities. As Heraclitus is reported to have said, in Plato's Cratylus dialogue, "you do not put your foot into the same river more than once". The key problem for human thought in this world of flux is survival. In this biological account of the origins of science, human thought plays cognitive and manipulative roles not completely dissimilar to those depicted by Soros. Memory plays a key role in economising on the effort needed to survive in the face of this Heraclitean flux, by imposing some order, or revealing disorder in, this external world. So, for example, memory allows recall of element types such as a "red" colour, and can associate this with "fading of light" in a "not-completely occluded sky" to allow a "red sunset" to be recognised, and maybe distinguished from the "red" associated with, say, a meteorite collision. By using conventions or classifications, memory can pave the way for science by distilling regularities from less recurrent phenomena. So the human memory is in effect producing low-level theories to aid comprehension of the flux of otherwise unique phenomena.

Science, according to Mach, proceeds by applying an epistemological economy principle Mach took the term "okonomisch" from discussions with a friend, Hermann, who was a political economist - to the ontological classifications or associations that are now treated as "facts". So, for example, Snel's law is an attempt to provide an economical rule covering different types of light refraction. "It is the object of science to replace, or save, experiences by the reproduction and anticipation of facts in thought ... science itself ... may be regarded as a minimal problem, consisting of the completest possible presentment of facts with the least possible expenditure of thought" [2, pp. 577, 586]. This allies the "entities are not to be multiplied without need" common sense of Ockham's razor to "the completest possible presentment of facts". The distinction between theories and facts is labile, higher-level theories treating lover-level theories as facts.

In this Machian account of science the physical, as well as the social, world is interpreted by human thought as an aid to survival. In evolutionary terms, theories that do not provide 
a good "reproduction and anticipation of facts in thought" will not be conducive to survival. This is the problem facing mainstream economic theories since the beginning of the financial crisis in 2007, and the subsequent great recession. Are the mainstream theories fit to survive? It is difficult to disagree with Soros that the answer to this question is "no".

It is not too difficult to find examples where thought in the physical sciences plays a manipulative function. Theories of DNA paved the way for genetic manipulation, changing the reality to be explained. Theories of human-made global warming have stimulated agreements to curb $\mathrm{CO}_{2}$ emissions, precisely with the aim of changing the world to be explained. A Gestalt view of the physical sciences takes into account technology as well. Sometimes the technological manipulation comes before the theory, as in the case of heat engines and modifications to the laws of thermodynamics. On other occasions the theories stimulate the technology, as in theories regarding sub-atomic particles and the making of nuclear weaponry and power stations. A step outside the laboratory reveals more of the reflexive two-way interaction between the cognitive and manipulative aspects of thought that the Soros guillotine takes to be confined to social sciences such as economics.

\section{The Duhem-Quine Problem}

The preceding section cast doubt on whether reflexivity is a key feature serving to separate physical sciences from social sciences such as economics. In this section we discuss the sketch Soros gives of a methodological approach that is appropriate to economics. This is based on analysing economic systems as composed of market participants who use Popper's logic of scientific discovery to learn from their mistakes. "As a market participant I formulate conjectures and expose them to refutation. I also assume that other market participants are doing the same thing whether they realize it or not. Their expectations are usefully aggregated in market prices. I can therefore compare my own expectations with prevailing prices. When I see a divergence, I see a profit opportunity. The bigger the divergence, the bigger the opportunity. When the price behaviour contradicts my expectations I have to re-examine my hypothesis. If I find myself proven wrong, I take a loss; if I conclude that the market is wrong, I increase my bet, always taking into account the risk that I am bound to be wrong some of the time" [1, p.39]

Market participants attempting to be guided by the Soros vision of Popperian conjectures and refutations are likely to be haunted by the Duhem-Quine problem. A conjecture about the future market price is likely to be guided by conjunctions of hypotheses about various potential influences on the market price. If the actual market price produces a loss, in relation to the participant's conjecture, the refutation is unfocussed: it will not be possible for the participant to know for sure which of the constituent hypotheses underlying the conjecture is to blame.

It is worth quoting Quine's "Two Dogmas of Empiricism" essay at some length. "The totality of our so-called knowledge or beliefs, from the most casual matters of geography and history to the profoundest laws of atomic physics or even of pure mathematics and logic, is a man-made fabric which impinges on experience only along the edges. Or, to change the figure, total science is like a field of force whose boundary conditions are 
experience. A conflict with experience at the periphery occasions readjustments in the interior of the field. Truth values have to be redistributed over some of our statements. Revaluation of some statements entails re-evaluation of others, because of their logical interconnections - the logical laws being in turn simply certain further statements of the system, certain further elements of the field. Having re-evaluated one statement we must re-evaluate some others, which may be statements logically connected with the first or may be statements of logical connections themselves. But the total field is so undetermined by its boundary conditions, experience, that there is much latitude of choice as to what statements to re-evaluate in the light of any single contrary experience. No particular experiences are linked with any particular statements in the interior of the field, except indirectly through considerations affecting the field as a whole" [3, pp. 42-43].

As pointed out in an earlier paper [10], participants in financial markets in a Soros world are engaged in guesses about other participants' expectations or conjectures of the type depicted in Keynes' beauty contest analogy. Participants' conjectures about the conjectures of other participants are also likely to involve conjectures about how "relevant" economic news, and policy makers' responses to such news, will affect the conjectures of other participants. Some participants, in FX markets for example, will have access to private information stemming from the customer-direct orders with end-user clients, so believing themselves to have an advantage in the guessing game. Participants making large deals may also feel grounds for having a higher degree of belief that the market price will move to their advantage. Should a refutation occur, the market price having not moved favourably in relation to the price conjectured by a particular participant, what can be concluded? As Duhem, the co-originator of the Duhem-Quine thesis, pointed out, "the physicist can never subject an isolated hypothesis to experimental test, but only a whole group of hypotheses: when the experiment is in conflict with his predictions, what he learns is that at least one of the hypotheses constituting this group is unacceptable and ought to be modified; but the experiment does not designate which one should be changed" [11, p. 187]. And so with market participants.

\section{The El Farol Bar}

We would not disagree with the Soros view that the axiomatic approach of mainstream economic theory, a formalisation of metaphors drawn from Newtonian mechanics in the neoclassical revolution of the 1870s [12], has produced "an axiomatic system based on deductive logic, not empirical evidence... at least some of the postulates of economics, notably rational choice and rational expectations, are dictated by the desire to imitate Newtonian physics rather than real-world evidence" [1, p.19]. A paraphrase of the biblical "by their deeds shall ye know them" should suffice in the explanation of how humans make economic decisions. We also concur that it is not the lot of human beings to know the future, and that economic theory should take on board the fact that economic decisions are made in face of endemic uncertainty regarding not only the future, but also regarding the present knowledge and dispositions of other participants. In such an uncertain world, simple decision rules or heuristics tend to outperform more complex, state-contingent rules [22]. 
The question, then, is whether it is possible to say anything interesting about this Mammonite flux. Are there regularities, or irregularities, that can be classified in an economical manner? Or are we left with an impossibility theorem regarding economic science, there being no economical classifications, in the sense of Mach, to be found?

We also concur with Soros that a sensible point of departure is an approach based on observation and inductive learning. As Hume pointed out, there are no logical grounds to expect past regularities to recur. But knowledge of such regularities, and irregularities, might provide the best show of realism in town. In what follows we use Arthur's parable of attendance at the El Farol bar [4] to illustrate how the inductive learning problems faced by participants in economic systems might be modelled.

The Arthur parable relates to the El Farol bar in Santa Fe, New Mexico, in which Irish music is played on a Thursday evening. The situation facing the $\mathrm{N}=100$ people who might attend the bar on a particular Thursday is that space is limited: the evening is only enjoyable if the bar is not too crowded, defined as less than 60 people attending. There is no communication between the potential bar participants, attendance decisions are taken to be unaffected by a participant's previous visits, and the only information available concerns how many people attended the bar in previous weeks. The problem is that there is no deductive model available to guide the participant in the decision as to whether to attend the bar, if fewer than 60 people are expected to attend; or stay away, if 60 or more people are expected to attend. If procedures such as coin tossing are ruled out, inductive rules, based on the number of people attending the bar in previous weeks, can be used to form expectations of attendance in the coming week and guide the participant's decision on whether or not to go.

Interestingly, in relation to mainstream economic models in which a single rational expectation is held, any commonality in expectations will be defied by the decisions made on the basis of that expectation. If all participants expect the bar to be too crowded, their decisions will lead the bar to be uncrowded; and vice versa. In the extreme this could lead to a flipping between 0 and 100 in bar attendance. Instead, Arthur postulates that participants use a variety of inductive conjectures about the coming week's bar attendance: using just the last bar attendance information; moving averages of past bar attendances; and so on. In response to the revealed bar attendance, participants revise their expectations and move away from errant predictors. "I believe that as humans in these contexts we use inductive reasoning: we induce a variety of working hypotheses, act upon the most credible, and replace hypotheses with new ones if they cease to work. Such reasoning can be modelled in a variety of ways. Usually this leads to a rich psychological world in which agents' ideas or mental models compete for survival against other agents' ideas or mental models - a world that is both evolutionary and complex" [4, p. 411].

In the original Arthur model, participants are in motion regarding their selection of inductive predictors of bar attendance, but attendance tends to average 60 and the predictors tend to settle on a 60/40 ratio between not-too-crowded and too-crowded forecasts. Various modifications can be made to the original Arthur specification of how bar attendance 
decisions are made. In [14] the participants take account of their own history of bar attendance, and their psychological reaction to their experiences. The number of times the participants enjoyed being at the bar because it was uncrowded, the occasions in which disappointment arose from the bar being too crowded, and the lost opportunities to have a good time when the participants did not go, but the bar was uncrowded, are taken into account. Habit formation, and thence hysteresis in bar attendance, is also introduced. Attempting to make the El Farol bar a more recognisable place by these modifications disturbs the 60/40 ecology. Hysteresis, for example, has a noticeable effect in increasing the periodicity in bar attendance.

It is interesting that applications of the El Farol parable to the behaviour of financial markets cite Soros, and adopt the term reflexivity to describe the processes involved in such models [13, p.37, footnote 16]. "Asset markets, we argue, have a recursive nature in that agents' expectations are formed on the basis of their anticipations of other agents' expectations, which precludes expectations being formed by deductive means. Instead, traders continuously hypothesize - continually explore - expectational models, buy or sell on the basis of those that perform best, and confirm or discard these according to their performance. Thus, individual beliefs or expectations become endogenous to the market, and constantly compete within an ecology of others' beliefs or expectations." [13, p.15] Soros does not mention such models in the essay under present consideration. It would be interesting to know whether or not he thinks that such models [15] incorporate his core ideas.

\section{Working Memory}

In the Machian account of science considered earlier, memory plays a key role in allowing humans to survive in the world of flux they inhabit, and paves the way for scientific classifications of the regularities, and irregularities, in this world. Psychologists and neuroscientists have distinguished various types of memory: short-term, long-term, episodic and semantic long-term, implicit and explicit, and working memory [6, Ch.1]. For Soros, "the complexity of the world in which we live exceeds our capacity to comprehend it ... we are obliged to resort to various methods of simplification: generalizations, dichotomies, metaphors, decision rules, and moral precepts, to mention just a few ... the brain is bombarded by millions of sensory impulses, but consciousness can process only seven or eight subjects concurrently ... the impulses need to be condensed, ordered, and interpreted under immense time pressure, and mistakes and distortions can't be avoided" [1, p.5].

The reference to "can process only seven or eight subjects concurrently" presumably relates to the working memory model [17], where the capacity limits have been estimated to be in the range of 5-9 pieces of transitory information that can be held concurrently in the brain and manipulated. The working memory is taken to be made up of a visuo-spatial sketch pad that stores information in a visual or spatial form for around 1-2 seconds; a phonological loop that allows the comprehension of spoken or written information; an episodic buffer that provides a link with long-term memory; and a central executive that performs a communication and coordination task [6, Ch.3]. 
What insights can the capacity limitations in working memory provide in relation to explaining the decisions made by participants in economic systems? These limitations would certainly seem to rule out the efficient markets hypothesis that the market prices of assets reflect all the information relevant to their determination. If participants can only hold less than 10 pieces of information in their working memories when making decisions, how is the full set of information to be reflected in market prices? There would have to be a substantial variation in the information sets used in different participants' working memories for the full information requirement to hold. Such variation is ruled out in the representative agent assumption that is often used to underpin the efficient markets hypothesis in mainstream economic models. The use of algorithms to design and execute trades can enhance the information sets used to inform trading decisions. Algorithms, however, have a survival problem in common with the non-algorithmic conjectures faced by humans. Even if an algorithm is used to choose between alternative algorithmic trading rules, a human has to choose the algorithm for algorithms. And that human will be faced with working memory capacity limits. The human eye operates by taking a small snapshot of the visual field, then jumping to another spot, and can perform only up to 5 such jumps per second [16, p.63]. This explains why traders typically operate with a small number of trading screens, often 4 , providing continuous information flows regarding the market in which they operate.

Soros argues that "market prices of financial assets do not accurately reflect their fundamental value... there are various pathways by which the mispricing of financial assets can affect the so-called fundamentals" [1, p.27]. If we take a Machian view of the world, the "underlying fundamentals" are a mosaic of elemental qualities in flux that are interpreted by the participants in economic systems. These interpretations are likely to differ, so the notion of "underlying fundamentals" has little or no meaning. All that market prices can reflect are the information sets used to shape active conjectures regarding market prices. Only in this very limited sense can markets be described as "efficient". To distinguish this world from that depicted in the Efficient Markets Hypothesis (EMH) requires a different terminology. We would suggest Contingent Markets Hypothesis $(\mathrm{CMH})$, where the only notion of "efficiency" is contingent on the varied and strictly limited information sets used by market participants when forming conjectures about the economic world.

Soros takes the Rational Expectations Hypothesis (REH) to imply "that there is a single correct set of expectations that people's views will converge around" [1, p.29]. In a $\mathrm{CMH}$ world it is "rational" for participants to use limited information sets to construct conjectures about the economic world, and use the feedback from events to strengthen or weaken their beliefs in these conjectures, replacing them as needed with different conjectures. It is "rational" to discard conjectures that are systematically wrong. The set of active conjectures will be in motion, and differ between participants. To distinguish this state of affairs from that depicted in the REH, we would suggest Rational Differentiated Conjectures Hypothesis (RDCH). 


\section{Far-From-Equilibrium}

In its current form, as applied to financial markets, Soros' theory lacks a mathematical or computational framework. The following agent-based market model, detailed in Lamba $[19,20]$, was not developed with reflexivity in mind. Rather it was constructed to demonstrate, as simply as possible, how the equilibrium solutions in economic and financial models lose stability when the standard assumptions are violated. Nonetheless it is based around elements that closely correspond to fallibility, both cognitive and manipulative functions, and positive feedback. As shown below, these can indeed combine to destabilise equilibria resulting in boom-bust, far-from-equilibrium, fat-tailed dynamics at realistic parameter values. Although the simple model presented below does not incorporate any specific notion of market fundamentals or underlying trends (but see the end of this Section), it reaches many of the same conclusions as Soros and may provide a stimulus for more accurate renderings of reflexivity.

Full details of the modeling assumptions can be found in $[19,20]$ but, briefly, only market participants (agents) whose opinions and strategies change over timescales of several days or longer, are directly simulated. At each time $t$, each agent is assumed to be in one of two states, either +1 or -1 , that roughly correspond to positive or negative opinions about the future value of an asset that are then disseminated and/or traded upon. Any given agent switches state relatively infrequently --- only when the current asset price $p(t)$ suddenly exceeds a threshold value defined by that agent's evolving expectations.

The average state of the agents is defined as the 'sentiment' $\sigma$ that can take values between -1 and +1 . Changes in the price $p(t)$ are due to both the arrival of new Brownian exogenous information $B(t)$ and changes in sentiment that are endogenous, i.e. generated within the market itself. Following [20], defining the log-price $r(t)=\ln p(t)$, and using the mathematical notation for differentials, we have

$$
d r=d B+\mathrm{k} d \sigma
$$

where $d r, d B$ and $d \sigma$ represent the changes in log-price, exogenous information and endogenous sentiment respectively. Larger values of the parameter $\mathrm{k}>0$ imply a larger influence of endogenous dynamics upon the asset price.

The correspondence with Soros reflexivity is as follows. The term $k d \sigma$ in the equation (1) plays the role of the manipulative (or participating) function. If it is absent then agents have no influence upon the system and the solution to (1) is just a price that follows the exogenously-determined (equilibrium) Brownian motion in accordance with the very strongest versions of the Efficient Markets Hypothesis (EMH). Next, the cognitive functions of the agents correspond to the evolving expectations (threshold dynamics) of each agent. The following point is mathematically subtle but crucial [19]. If these cognitive functions are sufficiently independent/uncorrelated with each other then agents' fallibilities will mostly cancel. This situation, which corresponds to small, essentially random, deviations of $\sigma$ around 0 agrees with the assumptions underlying the Rational Expectations Hypothesis 
(REH). And, once more, the term $\mathrm{k} d \sigma$ all-but-vanishes, leading to the equilibrium EMH Brownian pricing model. Soros correctly noted that "...without fallibility there would be no reflexivity." [1, p.4] but this observation should be strengthened to refer to systemic (i.e. non-cancelling) fallibility.

Thus in order to proceed we need to identify at least one form of fallibility that destroys the mathematical independence between agents' opinions/actions. This allows for the possibility of deviations from equilibrium that induce positive feedbacks. An obvious candidate is the phenomenon of herding, where agents in the minority opinion are fooled or pressured into joining the majority. Some likely causes of herding include psychological effects, momentum-trading strategies, or rational responses to perverse incentives [20] (such as the entirely understandable tendency for investment managers to 'chase the average' when their performance is being frequently judged against that of their peers).

It is a simple matter to introduce herding into the threshold dynamics. It is at this point that the reflexive nature of the model asserts itself - changes in the price and sentiment lead to changes in the threshold dynamics that lead to changes in the price and sentiment and so on. Estimates of the few critical parameters are easily established [20] and the results of a typical numerical simulation (with parameters chosen for asset rather than currency markets) are shown in Figure 1 and compared with the equilibrium Brownian price over a (hypothetical) 40 year period. Even low levels of herding destabilise the equilibrium solution causing boom-bust dynamics in $\sigma$, and hence in the price via (1). The unpredictable but rapid cascade processes that suddenly reverse long boom phases are described mathematically in [19] and can be explored interactively at [21].

The reluctance of mainstream economics to seriously consider far-from-equilibrium descriptions of economic reality, such as Soros reflexivity, is due in no small part to two fundamentally flawed (but mathematically expedient) assumptions about economic systems. The first is that equilibrating negative-feedbacks in economic systems operate near-instantaneously (as Soros points out in [1] this is a limiting case of his framework). However, the presence of participants with longer timescales, various forms of inertia/hysteresis/fallibility, and other limits to arbitrage can prevent this from happening. This delay in equilibration allows for positive-feedback effects to compete and sometimes dominate. Unfortunately such competition between positive and negative feedbacks can result in long periods that appear calm and predictable enough to be mistaken for a trend in the fundamentals of an equilibrium model.

However, even if equilibration were always instantaneous there is a second flaw that relates to economists' understanding of 'balance of forces'. In their rush to emulate physics they failed to appreciate that it is only in the simplest physical systems that a balance of forces implies the existence of a unique stable equilibrium, yet almost all neoclassical economics is imbued with this property. More complex systems (such as the above agent-based model or, say, a tectonic fault line) can support multiple internal configurations that correspond to different equilibria and gradual changes may cause unpredictable, sudden, and irreversible shifts between them. Thus the portion of reflexivity that considers the effects of mispricing 
on fundamentals might plausibly be restated in terms of mispricings causing the system's internal state to shift to a different configuration - one that persists even after the mispricing has been corrected. Applying this concept precisely enough to usefully model changes in, say, the credit and regulatory environments of a financial system would be a formidable but potentially very valuable undertaking.

\section{Concluding Remarks}

In the essay under review, George Soros provides a valuable exposure of the failings of mainstream theories in economics. On the negative side, we have argued that reflexivity does not provide a clear-cut distinction between a social science such as economics and the physical sciences; and that the Duhem-Quine thesis poses problems for a Popperian account of the way that participants might learn from refutations of conjectures, the latter being unfocussed because of the conjointness of the hypotheses underlying the conjectures. On a more constructive note, we have argued that models of inductive learning by participants who make decisions on the basis of strictly limited and heterogeneous information sets can capture the basic thrust of the Soros view of the economic world. The conjectures are in motion as the participants use the feedback from the revealed world to try and avoid those that are systematically in error. The Duhem-Quine problem means that there is no necessary reason why any participants who initially held the same conjecture would draw the same conclusion from the feedback provided by the experienced world.

Somewhat surprisingly, in view of the intractable uncertainties faced by participants in a Soros world, there is very little discussion of the emotional and biological influences on decision taking. The language of conjectures and learning from refutations tends to paint the participants in terms of their cognitive, reasoning and calculating capacities. In Smith's The Theory of Moral Sentiments, passions or emotions are the main drivers of economic behaviour, with reason intervening when reflection is made on behaviour in the way an impartial spectator might [18]. On financial markets making profits has been found to be associated with a rise in testosterone levels, higher volatility with enhanced cortisol [16]. Greed, fear, panic and euphoria are epithets often associated with financial markets. A more recognisable account of financial and other markets would incorporate the biological and emotional influences on the behaviour of the participants.

Finally, a mathematical model $[19,20]$ with close structural similarities to reflexivity affirms the significant consequences of reflexivity that have been postulated by Soros both for financial markets and for equilibrium-based thinking in economics.

\section{REFERENCES}

1. Soros, George (2013), "Fallibility, Reflexivity and the Human Uncertainty Principle", forthcoming, Journal of Economic Methodology. 
2. Mach, Ernst (1883), The Science of Mechanics, translated by T.J. McCormack. La Salle: Open Court Publishing, 1960.

3. Quine, Willard van Orman (1980), From a Logical Point of View. Cambridge MA: Harvard University Press

4. Arthur, W. Brian (1994), "Inductive Reasoning and Bounded Rationality", American Economic Review, 84.2, 406-411.

5. Cross, Rod, Grinfeld, Michael, Lamba, Harbir and Seaman, Tim (2005), "A Threshold Model of Investor Psychology", Physica A, 354, 463-478.

6. Baddeley, Alan D. (2004), Your Memory: a User's Guide. London, UK: Carlton Books.

7. Mach, Ernst (1897), The Analysis of Sensations, translated by C.M. Williams and S. Waterlow. New York: Dover Books, 1959.

8. Samuelson, Paul (1992), "My Life Philosophy: Policy Credos and Working Ways", in Michael Szenberg ed., Eminent Economists: Their Life Philosophies. New York: Cambridge University Press.

9. Cross, Rod (2006), "Paul Samuelson's Mach" in Michael Szenberg, Lall Ramrattan and Aron A. Gottesman eds., Samuelsonian Economics and the Twenty-First Century, pp. 330-341. New York: Oxford University Press

10. Cross, Rod and Strachan, Doug (1997), "On George Soros and Economic Analysis", Kyklos, 50.4, 561-574.

11. Duhem, Pierre, (1914), The Aim and Structure of Physical Theory, translated from the $2^{\text {nd }}$ French edition by Philip P. Wiener. New York: Atheneum, 1981.

12. Mirowski, Philip (1989), More Heat than Light, Cambridge UK: Cambridge University Press.

13. Arthur, W. Brian, Holland, John H., leBaron, Blake, Palmer, Richard and Tayler, Paul (1997), "Asset Pricing under Endogenous Expectations in an Artificial Stock Market", in W. Brian Arthur, Steven N. Durlauf and David A. Lane eds., The Economy as an Evolving Complex System II, Santa Fe Institute. Reading, MA: Addison-Wesley.

14. Cross, Rod, Grinfeld, Michael, Lamba, Harbir and Pittock, Ann (2005), "Frustration Minimization, Hysteresis and the El Farol Problem", in Michael P. Mortell, Robert E. O'Malley, Alexei Pakrovskii and Vladimir Sobolev eds., Singular Perturbations and Hysteresis. Philadelphia: Society for Industrial and Applied Mathematics.

15. Le Baron, Blake (2006), "Agent-Based Financial Markets: Matching Stylized Facts with Style", in David Colander ed., Post Walrasian Macroeconomics. New York: Cambridge University Press.

16. Coates, John (2012), The Hour between Dog and Wolf: Risk-Taking, Gut Feelings and the Biology of Boom and Bust. London, UK: Fourth Estate.

17. Baddeley, Alan D., and Hitch G. (1974), "Working Memory", in G.A. Bower ed., The Psychology of Learning and Motivation Vol 8, 47-89. New York: Academic Press.

18. Smith, Adam (1759), The Theory of Moral Sentiments. Oxford, UK: Oxford University Press, reprinted 1976.

19. Lamba, Harbir, "A queueing theory description of fat-tailed price returns in imperfect financial markets", European Physics Journal B77, 297-304 (2010).

20. Lamba, Harbir, "Implausible Equilibrium Solutions in Economics and Finance". Preprint, currently available at http://math.gmu.edu/ harbir/implausible.pdf . 
21. Lamba, Harbir, "Far-From-Equilibrium Dynamics: What if financial and economic equilibria are actually unstable? ". http://math.gmu.edu/ harbir/market.html .

22. Gigerenzer, Gerd (2013), Risk Savvy: How to make Good Decisions. New York: Penguin Books. 

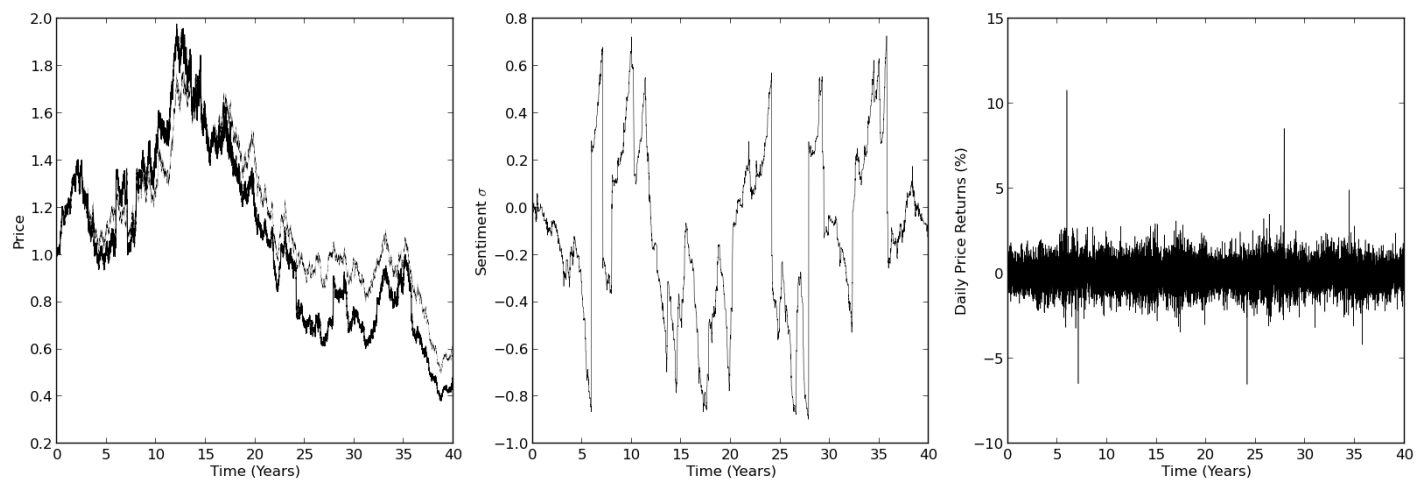

Figure 1: The left plot shows the output price of the model (thick line) and the Brownian equilibrium price (thin line) over a 40 year simulation. The difference between the two is caused by boom-bust endogenous effects that are approximately quantified by the deviations of $\sigma$ from 0 (middle plot). The right plot shows daily percentage price returns with the largest values corresponding to the sudden collapse of positive-feedback deviations. Periods of increased price activity and false tops and bottoms can be observed as the extended mispricings come to their very violent but unpredictable end (for the equilibrium solution there are no daily price changes exceeding $2 \%$ ). This minimal version of the model demonstrates that the unstable equilibrium solution is replaced by boom-bust dynamics and incorporating additional effects $[19,20]$ (such as the asymmetries caused by leverage and margin-calls) improves the fit with observed market statistics. 